\title{
Characteristic Mode Analysis of Unit Cells of Metal-Only Infinite Arrays
}

\author{
Yigit Haykir, Ozlem Aydin Civi
}

\author{
Department of Electrical and Electronics Engineering, \\ Middle East Technical University, Ankara, Turkey \\ *corresponding author, E-mail: yigit.haykir@metu.edu.tr
}

\begin{abstract}
Characteristic mode analysis of metal only unit cells of periodic structures is performed using Method of Moments based formulation. Ewald's transformation is incorporated for a fast and cost efficient solution and the advantages over spatial Green's function are discussed. The influence of the unit cell size on the characteristic modes is demonstrated. Various metal-only reflectarray elements are compared and their radiation characteristics are interpreted using the theory of characteristic modes. It is shown that characteristic modes of the unit cell can help us to understand the radiation and scattering behavior of the unit cell and this physical insight can be used in periodic array unit cell design.
\end{abstract}

\section{Introduction}

The theory of characteristic mode (TCM) was introduced by Garbacz [1], in 1971 and redefined by Harrington and Mautz $[2,3]$. Today, it is regarded as an efficient method for antenna design and analysis. Different varieties of antennas such as monopole and dipole antennas, mobile handset antennas and electrically small antennas have been analyzed using the TCM [4]. Their design aspects such as determination of the feeding position and coupling behaviors were thoroughly investigated [5-9]. Characteristic mode analysis is also suitable for the analysis of periodic arrays such as phased array antennas, transmitarray antennas, reflectarray antennas and frequency selective surfaces. In recent years, a reduced matrix solution based on modal basis functions is proposed for fast solution of the reflectarray antennas [10]. More recently, a microstip reflectarray unit cell has been analyzed using the characteristic modes and the modal reflection phases are discussed for the first time [11].

In this paper, characteristic mode analysis for metal-only array unit cell is demonstrated. A Galerkin moment method is utilized to analyze unit cell elements. Ewald's transformation [12] for periodic Green's function is performed for fast and efficient computation. Characteristic mode analysis is car- ried out by solving the corresponding generalized eigenvalue equation. This work focuses on the analysis of several periodic unit cell elements for reflectarray and frequency selective surface (FSS) applications. Frequency selective surfaces are optical filters in which element shape and the periodicity of elements determine the transmission characteristics [13]. Reflectarray antennas are made of periodic elements in varying shape and dimensions. They combine desired characteristics of both phased arrays and reflectors [14]. Phase distribution of the reflected field over the aperture of the array is adjusted by varying some parameter of the array element to obtain desired radiation characteristics. Although elements are usually printed on a dielectric substrate with one side metal cladding, metal-only solutions have also been investigated $[15,16]$. In this respect, our primary focus is on the characteristic mode analysis of metal-only unit cell elements.

In this work, a solution tool based on MoM has been developed for the characteristic mode analysis of metal-only unit cells in an infinite array environment. The relation between characteristic modes and the radiation characteristics is discussed. Preliminary results are presented in [17,18]. Formulation, results and discussion are extended in this paper.

The organization of this paper is as follows: Section 2 briefly explains the MoM formulation utilized for periodic unit cell. Periodic Green's function is defined. Ewald's transformation is briefly explained and the benefits are discussed. The details regarding the analysis of characteristic modes are presented. In Section 3, we touch upon the relation between the dimensions of the unit cell and the characteristic modes. Section 4 demonstrates an analysis for several reflectarray unit cell elements. Their reflection characteristics and characteristic modes are investigated. Section 5 presents concluding remarks as well as planned future work.

\section{Formulation}

The problem geometry is a 2-D infinite array of metallic structures with unit cell dimensions $a$ and $b$. Array is in $\mathrm{x}-\mathrm{y}$ plane. 
Total tangential electric field intensity on electric conductor surface becomes zero thus the following integral equation on the element surface is obtained

$$
\begin{aligned}
\hat{\boldsymbol{n}} \times \hat{\boldsymbol{n}} \times \boldsymbol{E}^{\boldsymbol{i}}=\hat{\boldsymbol{n}} \times \hat{\boldsymbol{n}} \times \\
\quad \frac{j \eta_{0}}{k_{0}} \int d \boldsymbol{r}^{\prime}\left[\nabla \nabla^{\prime} \cdot \boldsymbol{J}\left(\boldsymbol{r}^{\prime}\right)+k_{0}^{2} \boldsymbol{J}\left(\boldsymbol{r}^{\prime}\right)\right] G\left(\boldsymbol{r}, \boldsymbol{r}^{\prime}\right)
\end{aligned}
$$

where $\boldsymbol{E}^{i}$ is the incident field intensity, $\boldsymbol{J}$ is the current density to be solved, $G\left(\boldsymbol{r}, \boldsymbol{r}^{\prime}\right)$ is the Green's function of the system, $\eta_{0}$ and $k_{0}$ are intrinsic impedance and wavenumber, respectively. In this formulation, $e^{j \omega t}$ time dependence is assumed. Method of moments is applied to solve this problem. Current density is expanded using basis functions and then inner product with testing function is evaluated. Therefore linear equations are constructed in the form of matrix-vector multiplication, i.e., $\mathbf{Z}=\mathbf{I V}$. Moment matrix element is

$$
\begin{array}{r}
\mathbf{Z}_{\mathbf{m n}}=\frac{j}{\omega \epsilon_{0}} \int_{S m} d \boldsymbol{r} \boldsymbol{t}_{\boldsymbol{m}}(\boldsymbol{r}) \cdot \nabla \int_{S n} d \boldsymbol{r}^{\prime} \nabla^{\prime} \cdot \boldsymbol{b}_{\boldsymbol{n}}\left(\boldsymbol{r}^{\prime}\right) G\left(\boldsymbol{r}, \boldsymbol{r}^{\prime}\right)+ \\
j \omega \mu_{0} \int_{S m} d \boldsymbol{r} \boldsymbol{t}_{\boldsymbol{m}}(\boldsymbol{r}) \cdot \int_{S n} d \boldsymbol{r}^{\prime} \boldsymbol{b}_{\boldsymbol{n}}\left(\boldsymbol{r}^{\prime}\right) G\left(\boldsymbol{r}, \boldsymbol{r}^{\prime}\right)
\end{array}
$$

and the voltage vector element becomes

$$
\mathbf{V}_{\mathbf{m}}=\int_{S m} d \boldsymbol{r} t_{m}(\boldsymbol{r}) \cdot \boldsymbol{E}^{i}
$$

We use Galerkin method and choose both testing and basis functions as Rao-Wilton-Glisson (RWG) [19] functions. 3order Gaussian quadrature [20] is applied to solve integral equations with respect to source elements. Singularity extraction is applied when source and observation points are in vicinity, hence extracted terms are dealt using analytical representations as presented in [21].

Free space Green's function for 2-D periodicity can be written as

$$
G_{p}\left(\boldsymbol{r}, \boldsymbol{r}^{\prime}\right)=\frac{1}{4 \pi} \sum_{m=-\infty}^{\infty} \sum_{n=-\infty}^{\infty} e^{-j \boldsymbol{k}_{t \mathbf{0 0}} \cdot \boldsymbol{\rho}_{m n}} \frac{e^{-j k R_{m n}}}{R_{m n}}
$$

where

$$
\boldsymbol{k}_{\mathbf{t 0 0}}=\hat{\boldsymbol{x}} k_{0} \sin \theta_{i} \cos \phi_{i}+\hat{\boldsymbol{y}} k_{0} \sin \theta_{i} \sin \phi_{i} .
$$

The distance between source point and shifted observation points is

$$
R_{m n}=\left|\boldsymbol{R}-\boldsymbol{\rho}_{\boldsymbol{m n}}\right|=\left|\boldsymbol{r}-\boldsymbol{r}^{\prime}-\boldsymbol{\rho}_{\boldsymbol{m n}}\right|
$$

where the translation vector is $\boldsymbol{\rho}_{\boldsymbol{m} n}=m \boldsymbol{s}_{\mathbf{1}}+n \boldsymbol{s}_{\mathbf{2}}$ with $\boldsymbol{s}_{\mathbf{1}}$ and $s_{2}$ are vectors defining each unit lattice cell. Note that
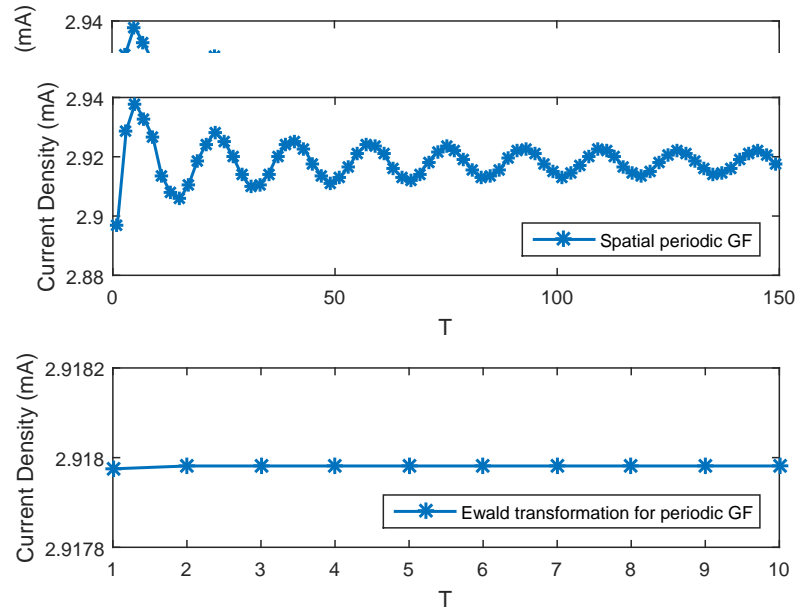

(b)

Figure 1: Maximum current density on $4 \mathrm{~mm}$ width square patch in $15.2 \mathrm{~mm}$ square unit lattice cell at $11 \mathrm{GHz}$, as a function of $T$. (a) Using spatial periodic Green's function. (b) Using Ewald's transformation.

$s_{1}=a \hat{\boldsymbol{x}}$ and $s_{2}=b \hat{\boldsymbol{y}}$, for a rectangle unit lattice cell with dimensions $a \times b$. Because there is infinite number of terms to be summed in (4), we need to truncate both summations. The problem here is that we need enormous number of terms to be calculated for an accurate solution.

In Fig. 1a, the convergence of the current density is illustrated at $11 \mathrm{GHz}$ for $4 \mathrm{~mm}$ width square patch constructed with 8 triangles and the dimension of the unit lattice cell is chosen as $15.2 \times 15.2 \mathrm{~mm}$. $T$ is the truncation number for the infinite double sum which limits the summations in (4) from $-T$ to $T$. As can be seen from the figure, calculating thousands of terms is not sufficient to solve an element with 8 triangles. For larger objects with more than hundreds of triangles, it will not be a feasible solution. In order to circumvent this pitfall, we have applied Ewald's transformation for the 3-D periodic Green's function. In this representation, Green's function is defined as a sum of modified spectral series and spatial series by utilizing complementary error function or Faddeeva function [22]. Note that modified spectral series are based on 2-D quasi-periodic Fourier series which are also known as Floquet series. Both spatial and spectral terms as well as their singularity-free expressions can be found in [22]. The aforementioned example is solved using this representation of the Green's function and the convergence of the current density is depicted in Fig. 1b. As can be seen, it converges at $T=2$ which circumvents a huge computational load. 
For the analysis of periodic slots/apertures on metallic plates, additional RWG elements should be defined to ensure the continuity of current at periodic unit cell boundary. For this reason, triangular meshes at the opposite sides of the unit cell boundary are constructed in mirror symmetry. Therefore, triangles coinciding with the right and left unit cell boundary as well as up and down unit cell boundary form additional RWG functions [23].

In the characteristic mode analysis, the moment matrix is divided into its real and imaginary components as $\mathbf{Z}=\mathbf{R}+j \mathbf{X}$ and since $\mathbf{R}$ is positive semi-definite, the generalized eigenvalue equation, i.e.,

$$
\mathbf{X} \mathbf{J}_{\mathbf{n}}=\lambda_{n} \mathbf{R} \mathbf{J}_{\mathbf{n}}
$$

yields orthogonal radiation patterns where $\mathbf{J}_{\mathbf{n}}$ represents eigenvector of the system and $\lambda$ is the corresponding eigenvalue. MATLAB's eig function is utilized for the solution of this equation. Using this method, we can decompose the current into its orthogonal components

$$
\mathbf{J}=\sum_{n} \alpha_{n} \mathbf{J}_{\mathbf{n}}
$$

in which each eigenvector is regarded as modal eigencurrents and independent of the excitation. Note that eigenvectors are normalized hence following orthogonality properties hold [4]:

$$
\begin{aligned}
& <\mathbf{J}_{\mathbf{m}}, \mathbf{R} \cdot \mathbf{J}_{\mathbf{n}}^{*}>=\delta_{m n} \\
& <\mathbf{J}_{\mathbf{m}}, \mathbf{X} \cdot \mathbf{J}_{\mathbf{n}}^{*}>=\lambda_{n} \delta_{m n} \\
& <\mathbf{J}_{\mathbf{m}}, \mathbf{Z} \cdot \mathbf{J}_{\mathbf{n}}^{*}>=\left(1+j \lambda_{n}\right) \delta_{m n}
\end{aligned}
$$

where $\delta_{m n}$ is 1 when $m=n$ and 0 , otherwise. The modal weighting coefficients in (7) can be found by taking inner product excitation with eigencurrents and applying orthogonality properties, i.e.,

$$
\alpha_{n}=\frac{<\boldsymbol{E}^{\boldsymbol{i}}, \mathbf{J}_{\mathbf{n}}>}{1+j \lambda_{n}} .
$$

The eigenvalues can be shown in a compact form, and called as modal significance (MS), in which values vary between 0 and 1 ,

$$
\mathrm{MS}_{n}=\frac{1}{\left|1+j \lambda_{n}\right|} .
$$

Therefore physical behavior of eigenvalues can be interpreted in terms of the modal significance values. Recently, a benchmarking work is performed by the Special Interest Group [24] for validation of characteristic mode solvers. We have participated in this work, and results show that our solver is in strong agreement with other participants' solver tools [25].

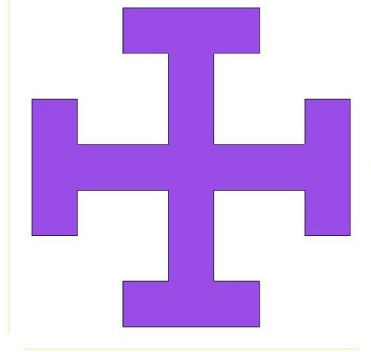

(a)

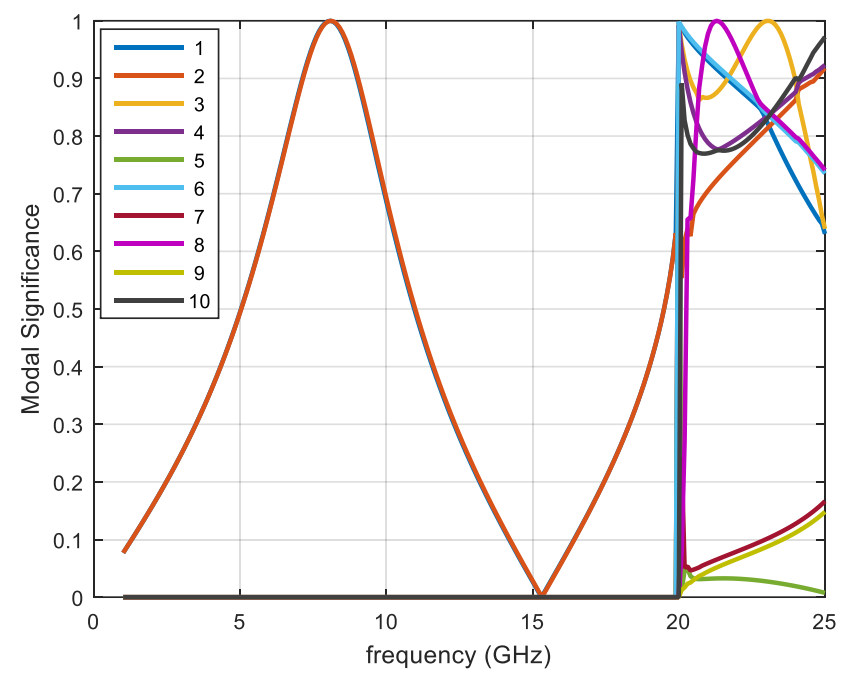

(b)

Figure 2: Jerusalem Cross element inside 15 x $15 \mathrm{~mm}$ square unit cell. (a) The geometry. (b) The modal significance values.

\section{Characteristic Modes on Array Unit Cells}

Jerusalem cross is a widely used element in FFS applications. In this work, we consider Jerusalem cross element, inside square unit cell with $15 \mathrm{~mm}$ edge size, as depited in Fig. 2a. The element geometry is taken from [26]. Characteristic modes of this unit cell is calculated using the formulation in Section 2. In Fig. 2b, modal significance values are shown as a function of the frequency. As can be seen from the geometry, only the first two modes are excited below $20 \mathrm{GHz}$. Since modal significance values are same, these modes can be regarded as degenerated modes. In other words, modes yield same eigenvalues but corresponding currents are $90^{\circ}$ out of phase. At frequencies higher than $20 \mathrm{GHz}$, higher order modes are excited as well and behaviors of the first two modes are changed. When frequency is below $20 \mathrm{GHz}$, the wavelength 


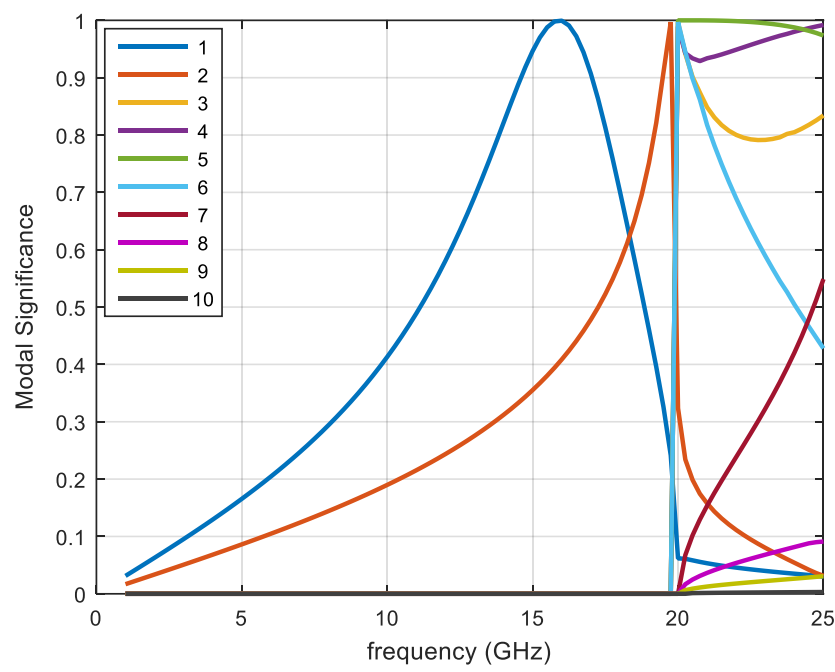

Figure 3: The modal significance values for rectangle patch inside 15 x $15 \mathrm{~mm}$ square unit cell.

is greater than $15 \mathrm{~mm}$, which is the cell size of the unit cell. Therefore, only first two characteristic modes are significant when cell size is smaller than wavelength. We can interpret this phenomena using Floquet series representation. When the cell size is smaller than one wavelength and the normal incidence is considered, only the first two Floquet modes are propagating. Therefore the frequency that makes the cell size one wavelength is a cut-off frequency for higher order modes. As a second example, a rectangle element is investigated inside $15 \times 15 \mathrm{~mm}$ square lattice cell. The rectangle shape of patch ensures that the element is not symmetric with respect to the center of the unit cell. As illustrated in Fig. 3, two significant modes do not yield same eigenvalues, in other words, they are no longer degenerate modes. Furthermore, $4 \mathrm{~cm} \mathrm{x} 6 \mathrm{~cm}$ rectangle patch element is placed in $6 \mathrm{~cm} \times 8 \mathrm{~cm}$ rectangle unit cell, and generated modal significance values are plotted in Fig. 4. This time, there are two non-zero MS values below $3.75 \mathrm{GHz}$. Between $3.75 \mathrm{GHz}$ and $5 \mathrm{GHz}$, we observe six non-zero MS values. At frequencies above $5 \mathrm{GHz}$, more higher modes are excited. We can conclude that $3.75 \mathrm{GHz}$ and $5 \mathrm{GHz}$ are cut-off frequencies due to the edge lengths of the lattice cell, $8 \mathrm{~cm}$ and $6 \mathrm{~cm}$, respectively.

\section{Metal-Only Reflectarray Unit Cell}

After examining modal characteristics of unit cells of different shapes, characteristic mode analysis is utilized to investigate reflection phase characteristics of metal only reflectarray unit cells. Three metal only unit cell geometries are chosen from

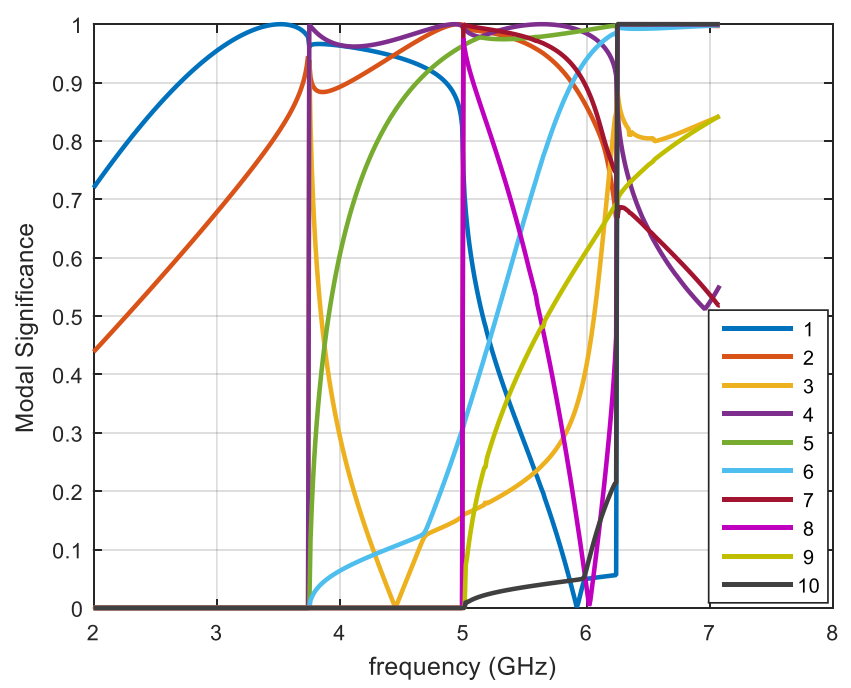

Figure 4: The modal significance values for rectangle patch inside $8 \times 6 \mathrm{~cm}$ rectangle unit cell.

the literature [16]: square-slot, rectangle-slot and square-slot element with square ring inside. In order to design a reflectarray, phase of the reflected field of unit cell is calculated as a function of control parameter of the reflectarray element, such as patch size, stub length. For the design of reflectarray, $360^{\circ}$ reflection phase range must be achievable with the unit cell by changing some parameter of the element geometry. Furthermore, reflection phase curve must be smoothly varying with the control parameter. Thus, our aim is to interpret modal behavior of reflectarray cells, get some physical insight and investigate the relation between behavior of characteristic modes and reflection phase curves; then use this information in the design of reflectarrays.

The first reflectarray element is an $\mathrm{L} \times \mathrm{L} \mathrm{mm}$ square-slot as depicted in Fig. 5a. The phase of the reflected field from reflectarray surface is calculated and presented in Fig. 5b, as a function of the slot size for various heights from the ground plane at $12.5 \mathrm{GHz}$. The phase of the reflected field is also solved in FEKO EM solver and strong aggreement is observed. As also illustrated in [16], the phase range increases as the distance between the ground plane and element increases. For instance when the distance increases from $4 \mathrm{~mm}$ to $10 \mathrm{~mm}$, the phase coverage increases about $200^{\circ}$. Characteristic mode analysis of this structure is performed using the proposed formulation in Section 2. In Fig. 6, corresponding modal significance values are calculated. It shows that only first four modes are excited where the first two modes and the next two modes are degenerated modes. When the distance between ground 


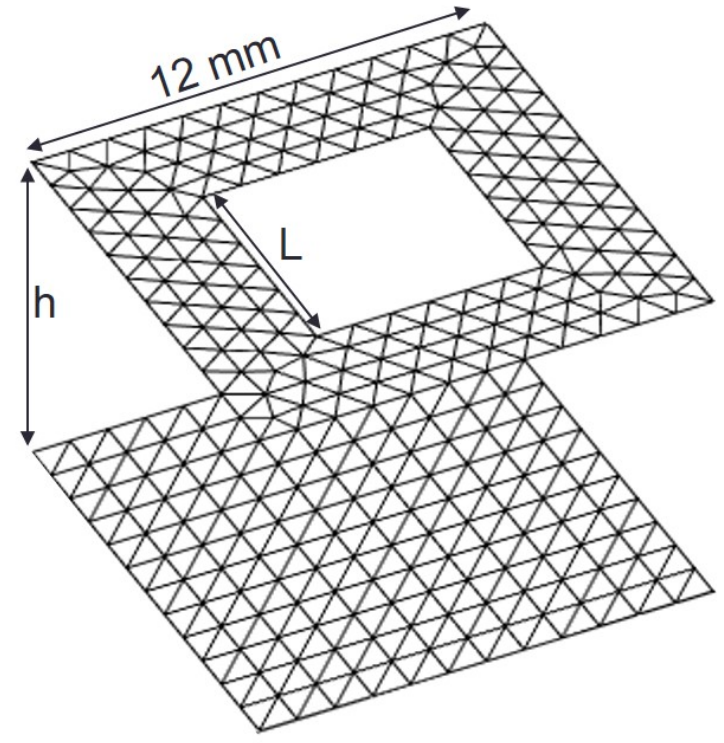

(a)

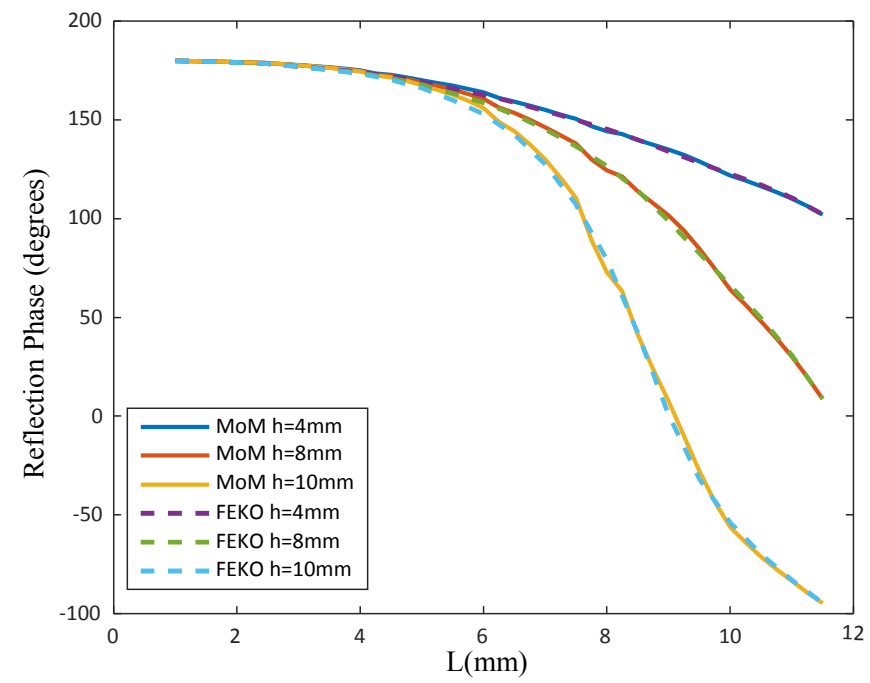

(b)

Figure 5: Square-slot reflectarray element. (a) The geometry and mesh. (b) The reflection phase.

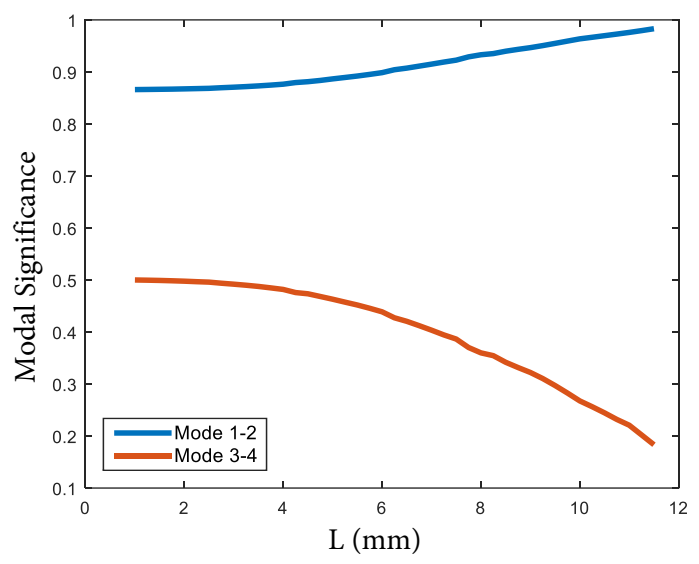

(a)

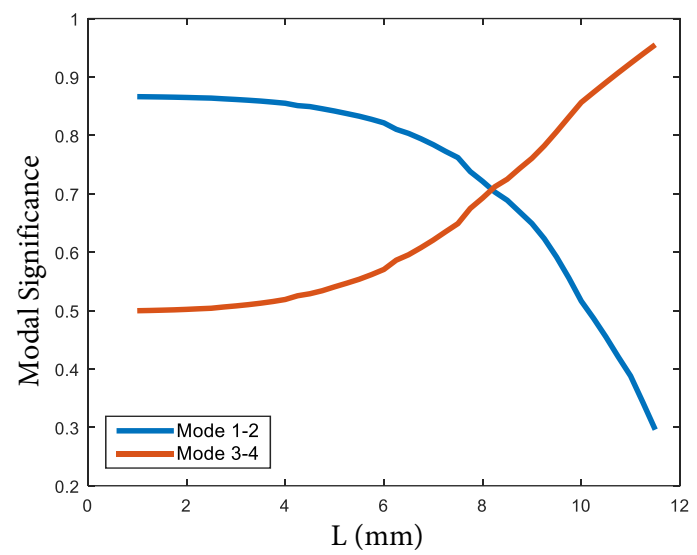

(b)

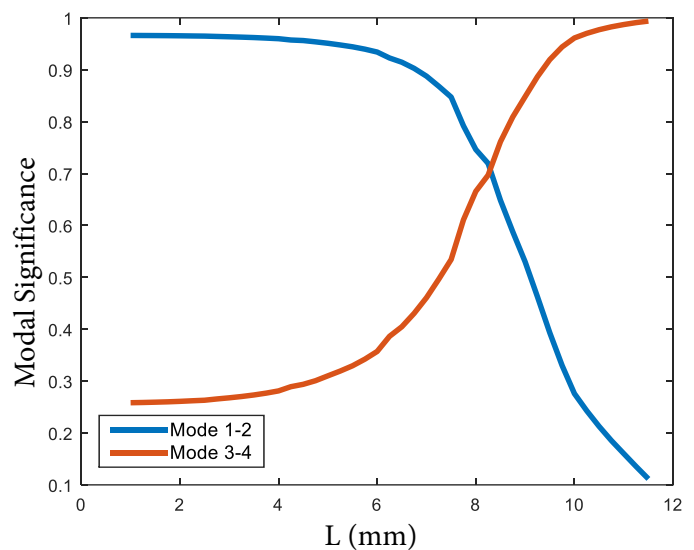

(c)

Figure 6: Modal significance for square-slot reflectarray elements. (a) h=4. (b) $h=8 \mathrm{~mm}$. (c) $\mathrm{h}=10 \mathrm{~mm}$. 


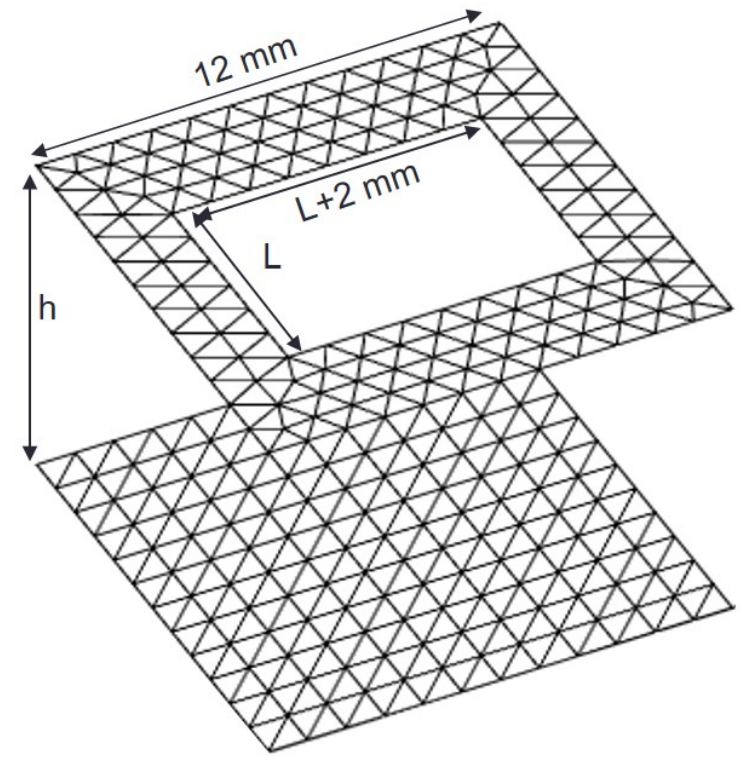

Figure 7: Rectangle-slot reflectarray element geometry and mesh.

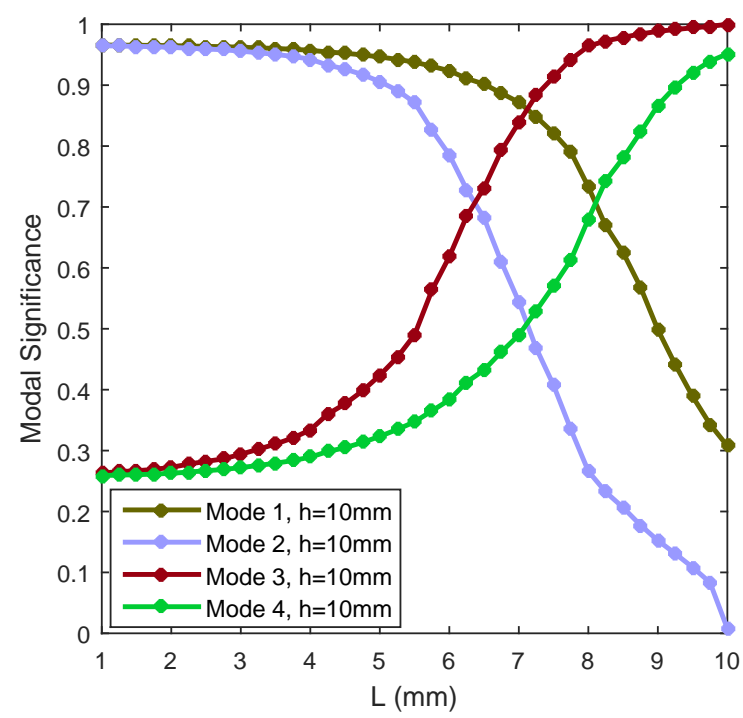

Figure 8: Modal significance for rectangle-slot reflectarray element.

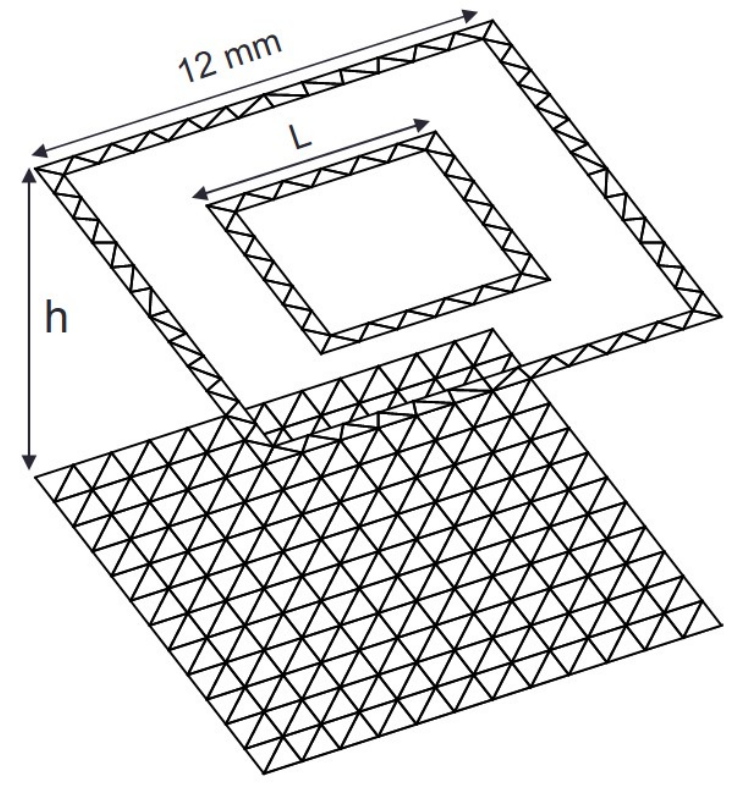

(a)

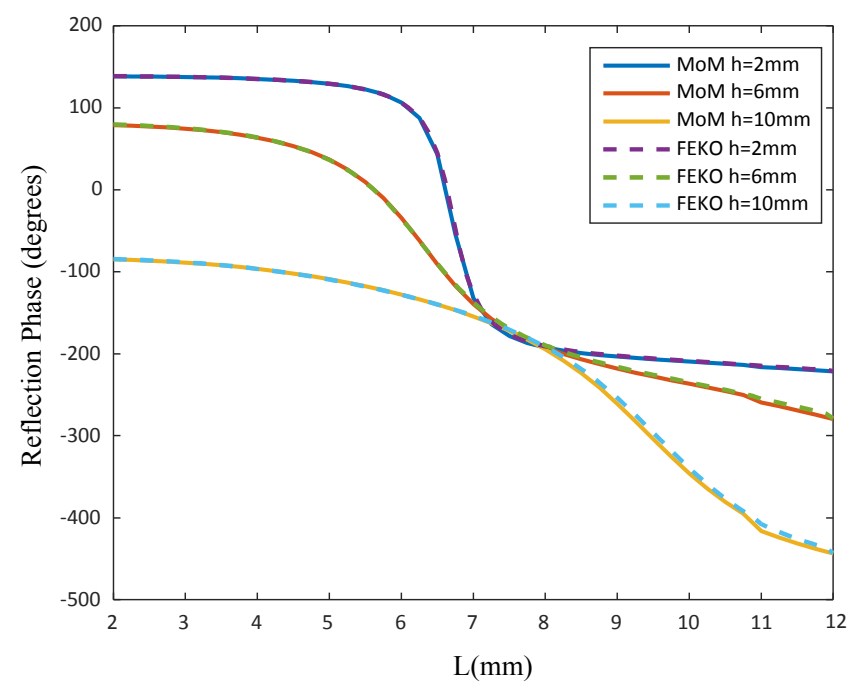

(b)

Figure 9: Ring-type reflectarray element in square-slot. (a) The geometry and mesh. (b) The reflection phase. 


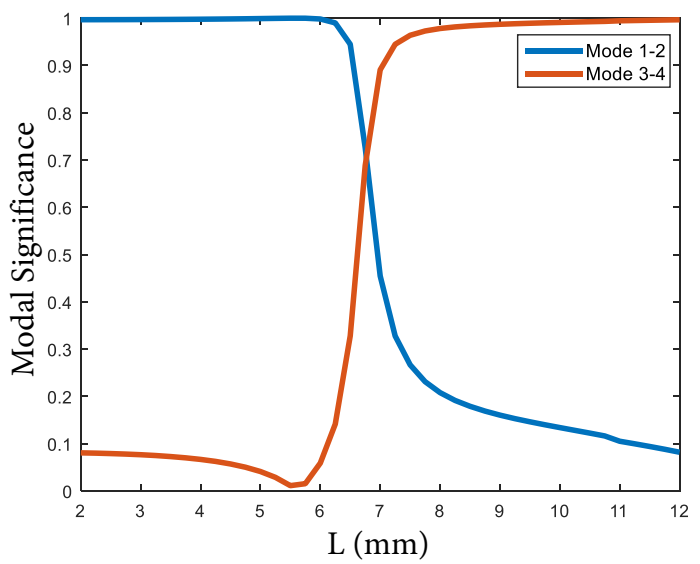

(a)

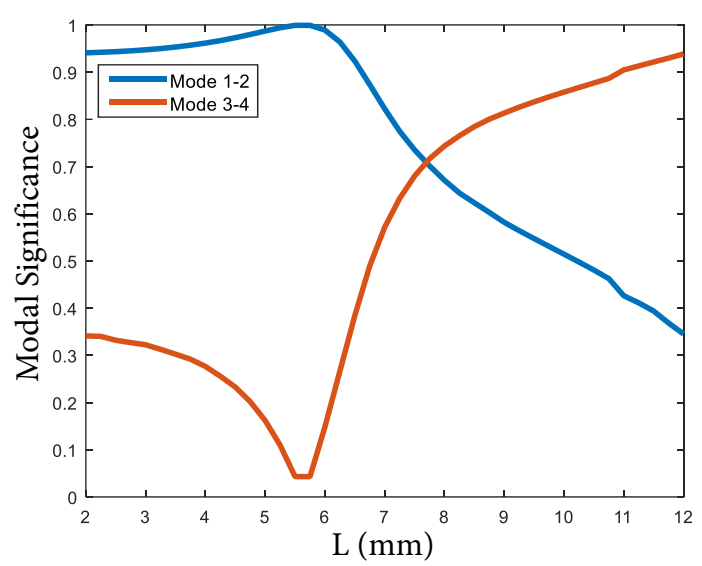

(b)

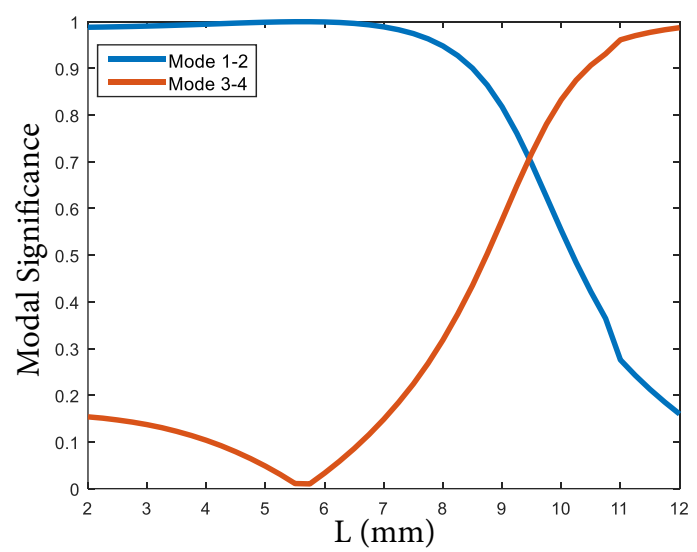

(c)

Figure 10: Modal significance for ring-type reflectarray elements. (a) $h=2 \mathrm{~mm}$. (b) $\mathrm{h}=6 \mathrm{~mm}$. (c) $\mathrm{h}=10 \mathrm{~mm}$. plane and the element increases, we observe more variation in modal significance values with $\mathrm{L}$. For instance, for $\mathrm{h}=4 \mathrm{~mm}$ modal significance of mode 1-2 remains close to 1 and MS of mode 3-4 changes from 0.5 to 0.2 ; whereas for case $h=10 \mathrm{~mm}$, modal significance (MS) of mode 1-2 decreases from 1 to 0.1 with an increase in L. While MS values of mode 1-2 decreases, second set of modes takes over i.e. MS values of mode 3-4 increases from 0.2 to 1 with $\mathrm{L}$. Therefore, we can conclude that large reflection phase ranges are related to large variation in the eigenvalues of at least two modes with the control parameter.

As a second example, rectangle slot of $\mathrm{L} \times(\mathrm{L}+2)$ is considered for the same configuration as depicted in Fig. 7. First two modes and next two modes are no more degenerated modes since the symmetry with respect to the origin of the unit cell is distorted. Slight separation of degenerated modes can be observed in Fig. 8. When the element is illuminated by an incidence field polarized in either horizontal or vertical directions with respect to the element, one of the first two modes and one of the next two modes would yield non-zero weighting coefficients in either square or rectangle slots. It means that the total current is formed by modal currents similar to the square case hence similar reflection characteristics are obtained for both rectangle and square slots. Due to this reason, reflection and modal characteristics are not presented in detail due to the brevity concerns.

Finally, we analyze $11 \times 11 \mathrm{~mm}$ square-slot with square ring inside at $12.5 \mathrm{GHz}$. The length for the outer edge of the ring is the varying parameter and the thickness of the ring is chosen as $1 \mathrm{~mm}$. Corresponding geometry is depicted in Fig. 9a. In Fig. 9b, $360^{\circ}$ phase coverage is observed. Again, our results closely follow the results extracted from FEKO EM solver. Corresponding modal significance values are plotted in Fig. 10. For heights $h=2 \mathrm{~mm}$ and $\mathrm{h}=6 \mathrm{~mm}$, it is observed from Fig. 9b that, reflection phase curves has a steep slope, which is not desirable for good reflectarray design. However, when $\mathrm{h}=10 \mathrm{~mm}$, reflection phase gradually changes with the size. Similar characteristics are observed in the variation of modal significance values of characteristic modes given in Fig. 10. More smooth variation in MS values as a function of $\mathrm{L}$ is obtained for the case $\mathrm{h}=10 \mathrm{~mm}$.

\section{Conclusions}

We have developed an efficient formulation to calculate characteristic modes for unit cell elements. Incorporation of Ewald transformation leads fast and a low-cost analysis. The relation between cell size and number of modes is discussed. Reflection and modal characteristics of different periodic unit cell elements are investigated, compared and presented. Investigation of the modal characteristics of the array unit cells provide 
physical insight which is valuable for the systematic and efficient designs of periodic structures such as array antennas, reflectarrays, frequency selective surfaces. In the future, this work can be extended for composite elements of metal and dielectrics. Reactive loadings for the reflectarray slot elements can also be analyzed. We believe that more information on radiation and scattering characteristic of large arrays can be extracted using the theory of characteristic modes.

\section{References}

[1] R. Garbacz and R. Turpin, "A generalized expansion for radiated and scattered fields," IEEE Transactions on Antennas and Propagation, vol. 19, no. 3, pp. 348-358, May 1971.

[2] R. Harrington and J. Mautz, "Theory of characteristic modes for conducting bodies," IEEE Transactions on Antennas and Propagation, vol. 19, no. 5, pp. 622-628, Sep 1971.

[3] — "Computation of characteristic modes for conducting bodies," IEEE Transactions on Antennas and Propagation, vol. 19, no. 5, pp. 629-639, Sep 1971.

[4] Y. Chen and C. Wang, Characteristic Modes: Theory and Applications in Antenna Engineering. Wiley, 2015.

[5] A. J. King and J. T. Bernhard, "Characteristic mode coupling in dipoles and dipole arrays," in 2015 9th European Conference on Antennas and Propagation (EuCAP), May 2015, pp. 1-3.

[6] M. Cabedo-Fabres, E. Antonino-Daviu, A. ValeroNogueira, and M. F. Bataller, "The theory of characteristic modes revisited: A contribution to the design of antennas for modern applications," IEEE Antennas and Propagation Magazine, vol. 49, no. 5, pp. 52-68, Oct 2007.

[7] A. A. Salih, Z. N. Chen, and K. Mouthaan, "Characteristic mode analysis and metasurface- based suppression of higher order modes of a $2 \times 2$ closely spaced phased array," IEEE Transactions on Antennas and Propagation, vol. 65, no. 3, pp. 1141-1150, March 2017.

[8] M. Khan and D. Chatterjee, "Characteristic mode analysis of a class of empirical design techniques for probefed, u-slot microstrip patch antennas," IEEE Transactions on Antennas and Propagation, vol. 64, no. 7, pp. 27582770, July 2016.
[9] Q. Wu, W. Su, Z. Li, and D. Su, "Reduction in out-ofband antenna coupling using characteristic mode analysis," IEEE Transactions on Antennas and Propagation, vol. 64, no. 7, pp. 2732-2742, July 2016.

[10] E. Ercil, L. Alatan, and O. A. Civi, "An efficient numerical solution method for reflectarrays of varying element sizes," IEEE Transactions on Antennas and Propagation, vol. 63, no. 12, pp. 5668-5676, Dec 2015.

[11] A. Maalik, R. G. Rojas, and R. J. Burkholder, "Characteristic modal decomposition of reflection phase of a microstrip-patch reflectarray unit-cell," in 2016 IEEE International Symposium on Antennas and Propagation (APSURSI), June 2016, pp. 425-426.

[12] K. E. Jordan, G. R. Richter, and P. Sheng, “An Efficient Numerical Evaluation of the Green's Function for the Helmholtz Operator on Periodic Structures," Journal of Computational Physics, vol. 63, pp. 222-235, Mar. 1986.

[13] B. Munk, Frequency Selective Surfaces: Theory and Design. Wiley, 2005.

[14] J. Huang and J. Encinar, Reflectarray Antennas, ser. IEEE Press Series on Electromagnetic Wave Theory. Wiley, 2007.

[15] W. An, S. Xu, and F. Yang, "A metal-only reflectarray antenna using slot-type elements," IEEE Antennas and Wireless Propagation Letters, vol. 13, pp. 1553-1556, 2014.

[16] R. Deng, F. Yang, S. Xu, and M. Li, “A low-cost metalonly reflectarray using modified slot-type phoenix element with $360^{\circ}$ phase coverage," IEEE Transactions on Antennas and Propagation, vol. 64, no. 4, pp. 15561560, April 2016.

[17] Y. Haykir and O. A. Civi, "Characteristic mode analysis of reflectarray unit cell," in 12th European Conference on Antennas and Propagation (EuCAP 2018), April 2018.

[18] Y. Haykir and O. A. Civi, "Periyodik birim hücresi karakteristik mod cözümlemesi (in Turkish)," in URSITÜRKIYE'2018 IX. Bilimsel Kongresi, Konya, September 2018.

[19] S. Rao, D. Wilton, and A. Glisson, "Electromagnetic scattering by surfaces of arbitrary shape," IEEE Transactions on Antennas and Propagation, vol. 30, no. 3, pp. 409-418, May 1982. 
[20] D. A. Dunavant, "High degree efficient symmetrical gaussian quadrature rules for the triangle," International Journal for Numerical Methods in Engineering, vol. 21, no. 6, pp. 1129-1148, 1985.

[21] D. Wilton, S. Rao, A. Glisson, D. Schaubert, O. AlBundak, and C. Butler, "Potential integrals for uniform and linear source distributions on polygonal and polyhedral domains," IEEE Transactions on Antennas and Propagation, vol. 32, no. 3, pp. 276-281, Mar 1984.

[22] P. Jorna, V. Lancellotti, and M. C. van Beurden, "Computational aspects of 2D-quasi-periodic-Green-function computations for scattering by dielectric objects via surface integral equations," Progress In Electromagnetics Research B, vol. 63, pp. 49-66, 2015.

[23] J.X. Su, X.W. Xu, M. He, K. Zhang, "Integral-equation analysis of frequency selective surfaces using ewald transformation and lattice symmetry," Progress In Electromagnetics Research, vol. 121, pp. 249-269, 2011.

[24] Website: http://www.characteristicmodes.org, 2018.

[25] Y. Chen et.al., "Benchmark problem definition and crossvalidation for characteristic mode solvers," in 12th European Conference on Antennas and Propagation (EuCAP 2018), April 2018.

[26] I. Stevanovic, P. Crespo-Valero, K. Blagovic, F. Bongard, and J. R. Mosig, "Integral-equation analysis of 3-D metallic objects arranged in 2-D lattices using the ewald transformation," IEEE Transactions on Microwave Theory and Techniques, vol. 54, no. 10, pp. 3688-3697, Oct 2006. 